https://helda.helsinki.fi

\title{
Phototropic Multiresponsive Active Nanogels
}

\section{Otsuka, Issei}

2019-12

Otsuka , I , Zhang , X \& Winnik , F M 2019 , ' Phototropic Multiresponsive Active Nanogels ' , Macromolecular Rapid Communications, vol. 40 , no. 24 , 1900479 . https://doi.org/10.1002/marc.201900479

http://hdl.handle.net/10138/321224

https://doi.org/10.1002/marc.201900479

acceptedVersion

Downloaded from Helda, University of Helsinki institutional repository.

This is an electronic reprint of the original article.

This reprint may differ from the original in pagination and typographic detail.

Please cite the original version. 


\section{WILEY-VCH}

Phototropic Multiresponsive Active Nanogels

Issei Otsuka, * Xuewei Zhang, and Françoise M. Winnik*

Dr. I. Otsuka

Univ. Grenoble Alpes, CNRS, CERMAV, 38000 Grenoble, France

E-mail: issei.otsuka@cermav.cnrs.fr

Dr. I. Otsuka, Dr. X. Zhang, Prof. F. M. Winnik

Department of Chemistry, Université de Montréal, CP 6128, Succursale Centre Ville, Montreal, QC H3C 3J7, Canada

Prof. F. M. Winnik

Department of Chemistry,University of Helsinki, Helsinki, Finland

E-mail: francoise.winnik@helsinki.fi

Prof. F. M. Winnik

International Center for Materials Nanoarchitectonics (MANA), National Institute for Materials Science (NIMS), 1-1 Namiki, Tsukuba, Ibaraki 305-0044, Japan 


\section{WILEY-VCH}

Abstract: Aqueous dispersions of nanogels that respond to switches in environmental $\mathrm{pH}$ or/and temperature by changes in their hydrodynamic radius $\left(R_{\mathrm{h}}\right)$ and/or $\zeta$-potential are prepared by reversible addition-fragmentation chain transfer (RAFT) polymerization-induced thermal $\left(70{ }^{\circ} \mathrm{C}\right)$ self-assembly (PITSA) of $N$-isopropylacrylamide (NIPAM) in the presence of a poly(methacrylic acid) (PMAA)-substituted macromolecular chain transfer agent and a crosslinker. Photochromic spiropyran (SP) moieties are coupled to the carboxylic acid groups of the nanogels. Upon UV irradiation, the neutral SP isomerizes to the zwitterionic merocyanine (ME) form. Upon UV light irradiation, microgels formed by assembly of SP-nanogels undergo a collective motion toward the UV-light source.

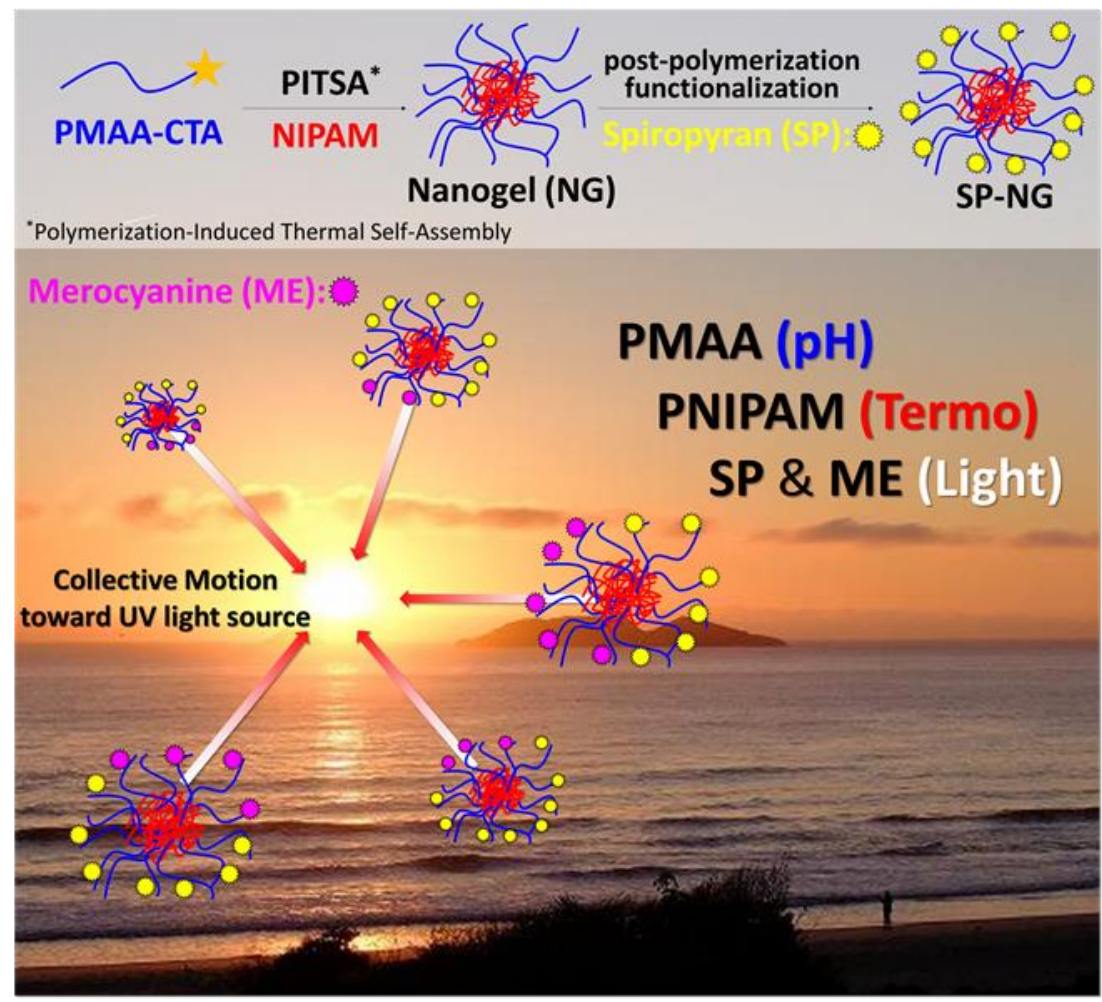




\section{WILEY-VCH}

The collective motion of flocks of birds, shoals of fish, bacterial colonies, or aggregates of live cells has fascinated biologists and physicists over the last decades in view of its complexity and beauty. It has inspired material scientists aiming to develop self-propelled objects moving collectively, known as "active matter". Active matter particles move by converting external energy into mechanical propulsion. ${ }^{[1,7]}$ Thus, Janus-Pt/Au nanorods dispersed in aqueous hydrogen peroxide are propelled by oxygen gas produced by the catalytic decomposition of hydrogen peroxide on the Pt surface of the nanorods. ${ }^{[3]}$ The fuels must be replenished to activate the continuous motion of these microrobots, which limits their practical implementation.

The application of energy in the form of light, ${ }^{[8-13]}$ heat, ${ }^{[14,15]}$ ultrasound, ${ }^{[16,17]}$ an electrical field, ${ }^{[18,19]}$ or magnetic induction ${ }^{[20,21]}$ can drive the collective motion of particles without refueling, in contrast to chemical reactions. ${ }^{[22,23]}$. Light is particularly attractive in this context, since it can be localized precisely in time and space and its energy content can be controlled through the choice of the irradiation wavelength. Light is used clinically in photodynamic therapy, opening the possibility for future therapeutic applications of active matter. Several studies have demonstrated the light induced directional control of active matter such as metal particles ${ }^{[13]}$, metal- ${ }^{[8,9]}$ or carbon- ${ }^{[10]}$ coated silica particles, and polymeric particles functionalized with photochromic dyes, ${ }^{[11,12]}$ such as azobenzenes ${ }^{[11]}$ and SP linked to hyperbranched polyester (Boltorn $\left.{ }^{\mathrm{TM}} \mathrm{H} 40\right) \cdot{ }^{[12]}$ The UV light-driven conversion of the nonionic SP to the zwitterionic ME creates an interfacial tension gradient on the surface of the particles from the "sunny" front side to the "dark" rear side such that the particles migrate collectively in the desired direction. Along their path, the particles may encounter $\mathrm{pH}$ gradients or changes in temperature, which could be recognized by the particles as signals to alter their morphology, to release their cargo, or to disintegrate, depending on the situation. Hence, there is an urgent need for multiresponsive active particles.

We report here waterborne nanogels $(\mathrm{NG}, \sim 80 \mathrm{~nm}$ in diameter) and microgels that are responsive to three orthogonal stimuli: light, change in temperature, and change in $\mathrm{pH}$. Their 


\section{WILEY-VCH}

core consists of crosslinked poly-( $N$-isopropylacrylamide) (PNIPAM), a polymer that undergoes a heat-induced coil-to-globule transition in water at $\sim 32{ }^{\circ} \mathrm{C} .{ }^{[24]} \mathrm{NGs}$ were obtained by RAFT $^{[25]}$-PITSA ${ }^{[26]}$ polymerization of NIPAM in the presence of a poly(methacrylic acid) chain transfer agent (PMAA-CTA), a cross-linker ( $N, N^{\prime}$-methylenebis(acrylamide), MBAM), and an initiator (azobis(4-cyanopentanoic acid, ACPA). The polymerization medium was water kept at $70{ }^{\circ} \mathrm{C}$. The growing PNIPAM chains became insoluble in the polymerization medium as they reached a given length. They self-assembled into nanoparticles stabilized by PMAACTA and the reaction mixture became turbid. The polymerization and cross-linking of NIPAM proceeded within the nanoparticles. Macroscopic phase separation or gelation did not occur, confirming the formation of colloidally-stable nanogels. (Figure 1a and Scheme S2a). The polymerization was quenched and the NGs were purified by extensive dialysis against water and recovered by freeze-drying.

The carboxylic acid groups of the NGs served as reactive centers for esterification with 1-(2-hydroxyethyl)-3,3-dimethylindolino-6'-nitrobenzopyrylospiran (SP-OH) in the presence of 4-(4,6-dimethoxy-1,3,5-triazin-2-yl)-4-methylmorpholinium chloride (DMTMM) (Figure $1 \mathrm{~b}$ and Scheme $\mathrm{S} 2 \mathrm{~b}$ ). The band at $1753 \mathrm{~cm}^{-1}$ in the FT-IR spectrum of the SP-NGs confirms the success of the esterification (Figure 2b), while the band at $1710 \mathrm{~cm}^{-1}$ corresponds to unreacted carboxylic acids. A weak multiplet $\left(8.2,8.0,6.5\right.$ ppm (peaks $\mathrm{x}, \mathrm{y}$, and $\mathrm{z}$ ) in the ${ }^{1} \mathrm{H} \mathrm{NMR}$ spectrum of SP-NG shown in Figure 1e) indicates the presence of the aromatic SP protons.

The UV-visible absorption spectrum of pristine NGs presents a strong band centered $320 \mathrm{~nm}$ characteristic of the trithiocarbonate moiety (Figure $2 \mathrm{~b}$, dotted black spectrum). This band has a shoulder (320 nm-430 nm) in the spectrum of SP-NG-1 (black spectrum), signaling the presence of the chromene chromophore of the SP group linked to the NGs. ${ }^{[27,28]}$ The level of SP incorporation $\left(5.63 \times 10^{-5} \mathrm{~mol} \mathrm{~g}^{-1}\right)$ was determined from the absorbance of the SP-NGs at $385 \mathrm{~nm}$ (see supporting information) indicates that $7.6 \mathrm{~mol} \%$ of carboxylic acids of the PMAA moieties of NGs were esterified with SP-OH. The fact that only a fraction of the 


\section{WILEY-VCH}

carboxylic acid groups, presumably located on the NG/water interface, suggests that most carboxylic acid units undergo hydrogen bonding with amide groups of the PNIPAM moiety in the core of NGs and are not accessible. ${ }^{[29]}$ Upon irradiation at $365 \mathrm{~nm}$ the SP group is converted to its merocyanine (ME) isomer (see Figure 1c and Scheme 2b). The UV-vis spectrum of SP-ME (red spectrum) features a broad band centered at $562 \mathrm{~nm}$, due to the $\pi-\pi^{*}$ electronic transition of the extended $\pi$-orbital of the conjugated ME indoline/chromene. The SP to ME photoisomerization is observed visually by the conversion of colorless SP-NG solutions into purple ME-NG solutions (inset in Figure 2b), and also by the emission spectra (Figure 2c, see supporting information).

The size and charge of the NGs in phosphate buffered saline (PBS, pH 7.2) at $25^{\circ} \mathrm{C}$ were determined by light scattering and $\zeta$-potential measurements, respectively. Dynamic light scattering (DLS) yields the NG's hydrodynamic radius $\left(R_{\mathrm{h}}\right)$, while static light scattering gives access to the radius of gyration $\left(R_{\mathrm{g}}\right)$ and molar mass $\left(M_{\mathrm{w}, \mathrm{SLS}}\right)$ (Table S2, Figures $2 \mathrm{~d}$ and S4). The shape factor, $\rho=R_{g} / R_{h}$, of the NGs is $\sim 0.78$, a value indicative of a solid sphere morphology $\left(R_{g} / R_{h}\right.$ theory $\left.=0.778\right) .{ }^{[30]}$ The spherical morphology of NGs was confirmed by TEM observation (Figure 2e). The number averaged radii $\left(R_{\mathrm{n}, \mathrm{TEM}}\right)$ statistically calculated from the TEM images of the dry NGs $\left(R_{\mathrm{n}, \mathrm{TEM}}=14.6\right.$ and $28.1 \mathrm{~nm}$ for NG-1 and NG-2, respectively $)$ are smaller than the $R_{\mathrm{h}}$ of the NGs in PBS ( $R_{\mathrm{h}}=29.0$ and $38.1 \mathrm{~nm}$ for the NG-1 and NG-2, respectively) implying that the NGs are hydrated in aqueous media at $25^{\circ} \mathrm{C}$. The $\zeta$-potentials of the NGs are negative at $\mathrm{pH} 7.2$ (-16.1 and $-12.6 \mathrm{mV}$ for the NG-1 and NG-2, respectively, as listed in Table S4) in agreement with the presence of carboxylate groups on the NGs surface. The carboxylic acid groups $\left(p \mathrm{~K}_{\mathrm{aMAA}}=4.36^{[31]}\right)$ are expected to be deprotonated (anionic) in neutral conditions. The NGs maintained their spherical morphology after functionalization with SP, as shown in the TEM image of SP-NG-1 (Figure 2f). The $R_{\mathrm{h}}$ values of SP-NGs are slightly larger than those of 


\section{WILEY-VCH}

NGs $\left(R_{\mathrm{h}}=33.5\right.$ and $45.6 \mathrm{~nm}$ for SP-NG-1 and SP-NG-2, respectively, Table S5) probably due to the presence of the bulky SP groups.

Dilute dispersions of NG and SP-NG in PBS $\left(1 \mathrm{~g} \mathrm{~L}^{-1}\right)$ of $\mathrm{pH} 4.0,7.2$, and 10.0 were heated from $20{ }^{\circ} \mathrm{C}$ to $50{ }^{\circ} \mathrm{C}$ and monitored by turbidimetry, $\mathrm{pH}$-dependent $\zeta$-potential measurements, and DLS (Figures 3a, 3b, and 3c, respectively). The transmittance of NG solutions of $\mathrm{pH}=4.0$, decreases sharply upon heating past $27{ }^{\circ} \mathrm{C}, \quad\left(T_{\mathrm{cp}}\right.$, pH4.0 $=29.6{ }^{\circ} \mathrm{C}$. $)$, signaling the collapse/dehydration of PNIPAM chains and subsequent shrinkage of individual NGs. The temperature-dependent changes in NGs' $R_{\mathrm{h}}(\mathrm{pH}=7.2,10.0)$ are less pronounced and start at a slightly higher temperature $\left(T_{\mathrm{cp}, \mathrm{pH} 7.2}=32.6{ }^{\circ} \mathrm{C}\right.$ and $\left.T_{\mathrm{cp}, \mathrm{pH} 10.0}=32.3{ }^{\circ} \mathrm{C}\right)$. The carboxylates on the surface of NGs at $\mathrm{pH} 7.2$ and 10.0 stabilize NGs against aggregation, as observed also for aqueous solutions of end-carboxylated PNIPAM chains. ${ }^{[32]}$ The SP-NGs $\zeta$-potential is hardly affected by changes in suspension $\mathrm{pH}$, confirming that a large fraction of the surface carboxylic acid groups bear SP groups. Due to the shrinkage of NGs upon heating, the surface area of the NGs and SP-NGs decreases upon heating, while their surface charge density increases, as reflected by their $\zeta$-potentials values, which become more negative upon heating. Overall, our studies demonstrate that the NGs and SP-NGs size and surface charge are readily controlled externally by $\mathrm{pH}$ and temperature changes.

The transformation of the nonionic SP form to the zwitterionic ME form upon UV light irradiation changes the interfacial tension between SP-NGs and solvent, which is known to be a driving force for the light induced self-propelled collective motion of the SP-modified particles. ${ }^{[12]}$ We tested this property with SP-microgels obtained by assembly of SP-NG placed in close proximity in a concentrated $\left(5 \mathrm{~g} \mathrm{~L}^{-1}\right)$ colloidal SP-NG suspension in PBS of $\mathrm{pH} 7.2$ at $25^{\circ} \mathrm{C}$ (Figure S8). DLS profiles of the colloidal suspension confirmed the coexistence in the dispersion of microgels $\left(R_{\mathrm{h}} \sim 280 \mathrm{~nm}\right)$ and individual SP-NG $\left(R_{\mathrm{h}} \sim 35 \mathrm{~nm}\right)$ (Figure $\left.\mathrm{S} 7\right)$. 


\section{WILEY-VCH}

When a UV light beam $(\lambda=325-375 \mathrm{~nm})$ is focused onto a microgel suspension placed on the microscope sample holder, one can detect the motion of individual microgels in Brownian motion towards the UV light (Video S1). The light spot appears pink in the artificial color map of the UV light intensity distribution that was obtained by observing a UV-absorbing fluorescence reference glass slide under UV irradiation (Figures S9). Trajectories of moving microgels composed of sequences of 100 frames extracted from Video S1 are presented in Figure 4. The contrast and color of the pictures were adjusted by using ImageJ software in such a way that the color of the microgels gradually changes from dark blue to light yellow as the frame number increases. Although the motion of the microgels in the direction orthogonal to the focal plane cannot be evaluated, the majority of motile microgels followed a trajectory toward the UV light focus on the focal plane located at lower right side of the microscopic field (see Figure 4 comparing with Figure S9), indicating that the microgels moved collectively toward the UV light source. The longest straight trajectory of a motile microgel (green rectangle in Figure 4) was $c a .11 .5 \mu$ m per 100 frames, providing an estimated in-plane velocity of $c a$. $140 \mathrm{~nm} \mathrm{~s}^{-1}$.

Previous studies have reported the collective motion of SP-terminated hyperbranched polymers toward a UV light source and discussed the mechanism underlying their motion. ${ }^{[12]}$ A UV light ray that hits collides undergoes reflection, absorption, diffraction, and refraction. If the radius $(R)$ of the particle is comparable to the wavelength $(\lambda)$ of the light (size parameter $(s)$ $=2 \pi R / \lambda \cong 1$, i.e. Mie scattering) or if it is much larger than the wavelength of light (s $>1$, i.e. Fraunhofer scattering), the light is absorbed or refracted by the particle, resulting in a gradient distribution of the UV irradiation from the rear surface of the particle to the front. Given the microgels' $R_{\mathrm{h}}\left(R_{\mathrm{h}}=280 \mathrm{~nm}\right)$ and the wavelength of the UV light source $(\lambda=325-375 \mathrm{~nm})$, the size parameter here is $s=4.7-5.4(>1)$. Hence, it is reasonable to predict that there is a gradient distribution of UV irradiation on the surface of the microgels, resulting in a gradient distribution 


\section{WILEY-VCH}

of the ME isomers i.e. there is a larger number of ME isomers on the "sunny" side of the colloids, compared to the "dark" rear side. Consequently, the collective motion of the microgels is driven by a gradient of interfacial tension on the surface of the microgels due to the difference in polarity between of the SP and ME isomers. The interfacial tension in aqueous media of ME is lower than that of the SP isomer on the surface. Thus, the collective motion is directed toward the "sunny" side where the interfacial tension between the microgels and fluid should be lower. Further studies under different conditions and with other stimuli (solvents, temperature, $\mathrm{pH}$, etc.) are under investigation with a view on enhancing the collective motion of microgels.

In summary, stimuli-responsive SP-NGs were prepared by PITSA of NIPAM mediated

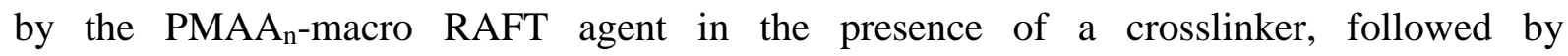
functionalization with an SP derivative. The $R_{\mathrm{h}}$ and $\zeta$-potentials of the SP-NGs were adjusted through changes in solution temperature and $\mathrm{pH}$. Irradiation of UV light onto aqueous dispersions of microgels composed of assembled SP-NG induces a gradient distribution of the photoisomerized ME groups on the surface of microgels from the "sunny" front side to the "dark" rear side, giving rise to a gradient of the interfacial tension on the colloid surfaces that drives the directed collective motion of the microgels toward the UV light source. The UV light-directed swimming colloids that have the multi-responsiveness reported here for the first time create opportunities for polymeric active matter to act as optically-guided artificial molecular motors in numerous fields.

\section{Experimental Section}

Observation of the UV-responsive Motion of the SP-NGs: A Nikon Eclipse TE2000U inverted epifluorescence optical microscope equipped with an Osram HBO 103W/2 mercury lamp light source and Dapi/Hoechst/AMCA filter cube (excitation filter: BP 350/50; dichroic mirror: 400 and Emission filter: 460/50) was used to observe the UV-responsive motion of the colloids. The averaged UV energy density was measured to be $241.5 \mu \mathrm{W} / \mathrm{cm}^{2}$ using Thorlabs 


\section{WILEY-VCH}

PM100D power meter. The artificial color map of the intensity distribution of the UV light

(Figure S9) was obtained by observing UV-absorbing fluorescence reference blue glass slide (Chroma Technology Co.) without specimen under the UV irradiation. About $1 \mathrm{~mL}$ of the colloidal solution was injected in a circular well (Fluorodish $35 \mathrm{~mm}$ diameter round flask with a $0.17 \mathrm{~mm}$ thickness glass bottom imaging zone of $23 \mathrm{~mm}$ in diameter). A $60 \times / 1.40$ oil objective (Nikon CFI Plan Apochromat DIC) was used for observing the motion in a microscopic field of $146 \mu \mathrm{m} \times 115 \mu \mathrm{m}$. The sample was illuminated by UV light focused by the objective. The light spot on the focal plane has a diameter of $c a .300 \mu \mathrm{m}$ as the field of illumination. Images of the motion were recorded as movies consisted of a sequence of pictures taken in bright field (transmitted light optics using a $12 \mathrm{~V}$ tungsten lamp, exposure time of each frame: $200 \mathrm{~ms}$ ) at a constant time interval ( $640 \mathrm{~ms}$ ) by a 12 bit CoolSnap fx CCD camera and by using Metamorph (Molecular Devices) and ImageJ (National Institutes of Health) software.

\section{Supporting Information}

Supporting Information is available from the Wiley Online Library or from the author.

\section{Acknowledgements}

This study was financially supported by Natural Sciences and Engineering Research Council of Canada and a grant "COOPERA" (Contract N¹49724) from la Région Rhône-Alpes, France and Ministry of Education, Culture, Sports, Science and Technology (MEXT), Japan, under their WPI Program. I.O. is grateful for CNRS and a grant "Explo'RA PRO" (Contract $N^{\circ} 149739$ ) from la Région Rhône-Alpes, France which supported his sabbatical research leave at Université de Montréal. The authors acknowledge for the support of C. Pellerin for FT-IR measurements, J. Niskanen for SEC-MALS measurements, A. Fouilen for TEM observations, and M. Vasseur for optical microscope observations.

Received: ((will be filled in by the editorial staff))

Revised: ((will be filled in by the editorial staff)) Published online: ((will be filled in by the editorial staff))

\section{References}

[1] M. C. Marchetti, J. F. Joanny, S. Ramaswamy, T. B. Liverpool, J. Prost, M. Rao, R. A. Simha, Reviews of Modern Physics 2013, 85, 1143.

[2] J. Zhang, E. Luijten, B. A. Grzybowski, S. Granick, Chem. Soc. Rev. 2017, 46, 5551. 


\section{WILEY-VCH}

[3] W. F. Paxton, K. C. Kistler, C. C. Olmeda, A. Sen, S. K. St. Angelo, Y. Cao, T. E. Mallouk, P. E. Lammert, V. H. Crespi, Journal of the American Chemical Society 2004, 126, 13424.

[4] I. Theurkauff, C. Cottin-Bizonne, J. Palacci, C. Ybert, L. Bocquet, Physical Review Letters 2012, 108, 268303.

[5] T. Sanchez, D. T. N. Chen, S. J. DeCamp, M. Heymann, Z. Dogic, Nature (London, U. K.) 2012, 491, 431.

[6] Y. Wu, Z. Wu, X. Lin, Q. He, J. Li, ACS Nano 2012, 6, 10910.

[7] M. Liu, L. Liu, W. Gao, M. Su, Y. Ge, L. Shi, H. Zhang, B. Dong, C. Y. Li, Nanoscale 2015, 7, 4949.

[8] I. Buttinoni, G. Volpe, F. Kümmel, G. Volpe, C. Bechinger, Journal of Physics: Condensed Matter 2012, 24, 284129.

[9] B. Dai, J. Wang, Z. Xiong, X. Zhan, W. Dai, C.-C. Li, S.-P. Feng, J. Tang, Nature Nanotechnology 2016, 11, 1087.

[10] C. Lozano, B. ten Hagen, H. Löwen, C. Bechinger, Nature Communications 2016, 7, 12828. [11] J.-P. Abid, M. Frigoli, R. Pansu, J. Szeftel, J. Zyss, C. Larpent, S. Brasselet, Langmuir 2011, 27, 7967.

[12] W. Li, X. Wu, H. Qin, Z. Zhao, H. Liu, Adv. Funct. Mater. 2016, 26, 3164.

[13] C. Chen, F. Mou, L. Xu, S. Wang, J. Guan, Z. Feng, Q. Wang, L. Kong, W. Li, J. Wang, Q. Zhang, Advanced Materials 2017, 29, 1603374.

[14] H.-R. Jiang, N. Yoshinaga, M. Sano, Physical Review Letters 2010, 105, 268302.

[15] H. Wang, Y. Liang, W. Gao, R. Dong, C. Wang, ACS Applied Materials \& Interfaces 2017, 9, 43211 .

[16] W. Wang, L. A. Castro, M. Hoyos, T. E. Mallouk, ACS Nano 2012, 6, 6122.

[17] K. J. Rao, F. Li, L. Meng, H. Zheng, F. Cai, W. Wang, Small 2015, 11, 2836.

[18] S. Gangwal, O. J. Cayre, M. Z. Bazant, O. D. Velev, Physical Review Letters 2008, 100, 058302.

[19] J. Yan, M. Han, J. Zhang, C. Xu, E. Luijten, S. Granick, Nature Materials 2016, 15, 1095. [20] J. Yan, M. Bloom, S. C. Bae, E. Luijten, S. Granick, Nature 2012, 491, 578.

[21] W. Gao, S. Sattayasamitsathit, K. M. Manesh, D. Weihs, J. Wang, Journal of the American Chemical Society 2010, 132, 14403.

[22] S. Das, A. Garg, A. I. Campbell, J. Howse, A. Sen, D. Velegol, R. Golestanian, S. J. Ebbens, Nature Communications 2015, 6, 8999.

[23] J. Simmchen, J. Katuri, W. E. Uspal, M. N. Popescu, M. Tasinkevych, S. Sánchez, Nature Communications 2016, 7, 10598.

[24] A. Halperin, M. Kröger, F. M. Winnik, Angewandte Chemie International Edition 2015, $54,15342$.

[25] S. Perrier, Macromolecules 2017, 50, 7433.

[26] C. A. Figg, A. Simula, K. A. Gebre, B. S. Tucker, D. M. Haddleton, B. S. Sumerlin, Chemical Science 2015, 6, 1230.

[27] N. W. Tyer, R. S. Becker, Journal of the American Chemical Society 1970, 92, 1289.

[28] M. Moniruzzaman, C. J. Sabey, G. F. Fernando, Polymer 2007, 48, 255.

[29] G. Chen, A. S. Hoffman, Nature 1995, 373, 49.

[30] G. S. Grest, L. J. Fetters, J. S. Huang, D. Richter, Adv. Chem. Phys. 1996, 94, 67.

[31] A. Katchalsky, G. Blauer, Transactions of the Faraday Society 1951, 47, 1360.

[32] P. A. FitzGerald, S. Gupta, K. Wood, S. Perrier, G. G. Warr, Langmuir 2014, 30, 7986. 


\section{WILEY-VCH}

(a)

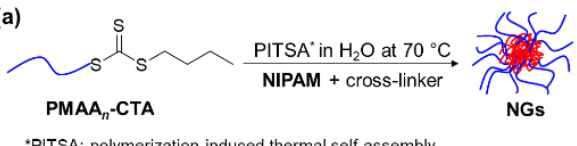

(b)

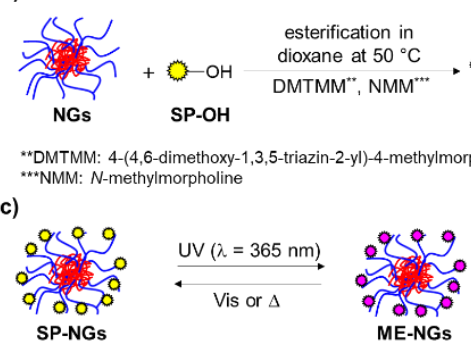

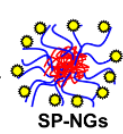

SP-NGs (d) NG-1

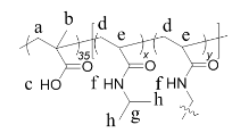

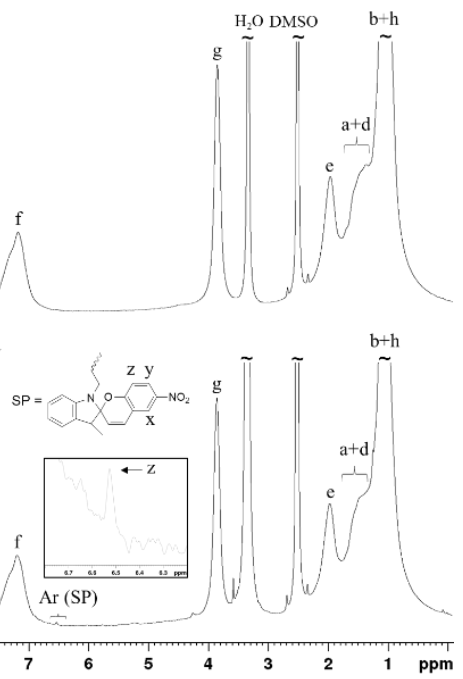

Figure 1. a) Synthesis of NGs via RAFT-PITSA. b) Post-polymerization functionalization of NGs with SP-OH via esterification. c) Photo-/thermal-induced isomerization between SP-NGs and ME-NGs. d) ${ }^{1} \mathrm{H}$ NMR spectrum of NG-1 in DMSO- $d_{6}$. e) ${ }^{1} \mathrm{H}$ NMR spectrum of SP-NG-1 in DMSO- $d_{6}$. 


\section{WILEY-VCH}

(a)

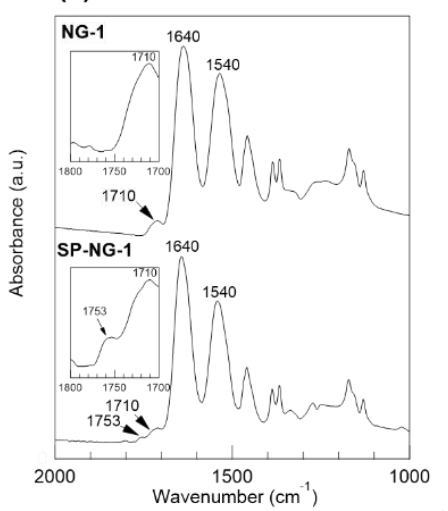

(d)

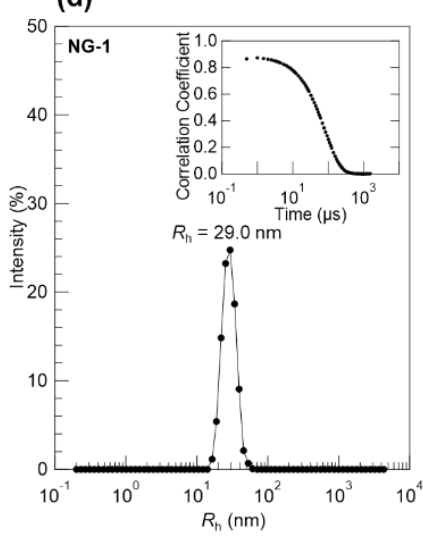

(b)

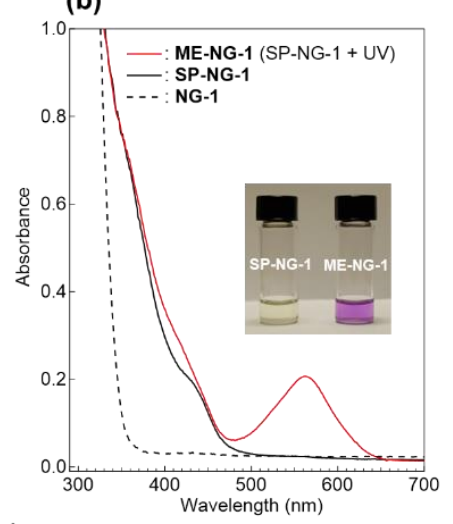

(e)

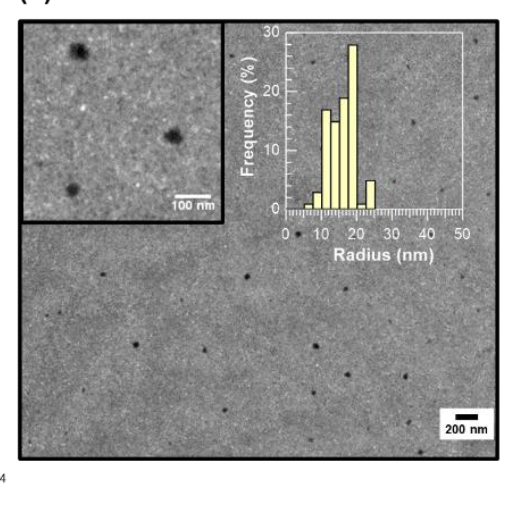

(c)

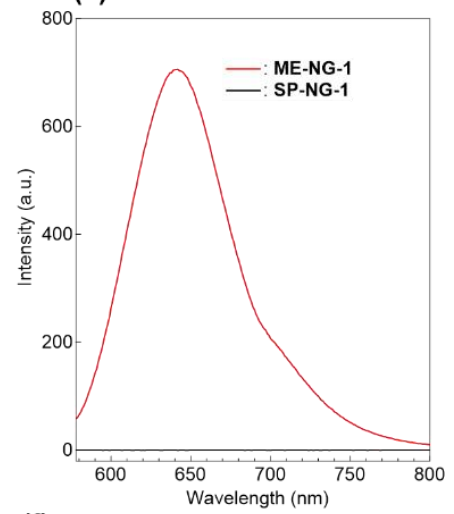

(f)

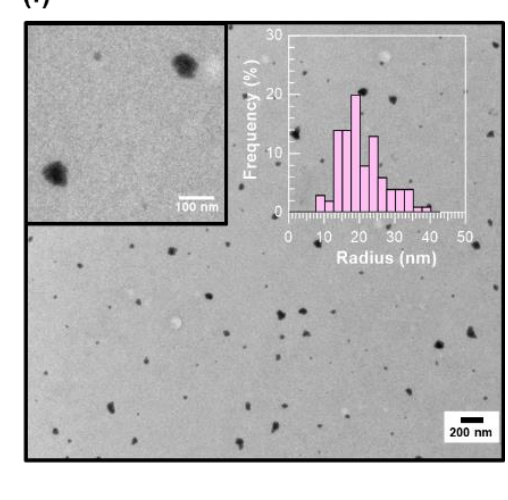

Figure 2. a) FT-IR spectra of NG-1 (top) and SP-NG-1 (bottom) at 1000-2000 cm-1. Inserts are their zoomed spectra at $1700-1800 \mathrm{~cm}^{-1}$. b) UV-vis absorption spectra of NG-1 (dotted black), SP-NG-1 (black), and ME-NG-1 (red) in DMSO (1 $\left.\mathrm{g} \mathrm{L}^{-1}\right)$ with a $10 \mathrm{~mm}$ optical path length. Insert is a picture of the solutions of SP-NG-1 and ME-NG-1 in DMSO. c) Emission spectra (excitation wavelength $\left(\lambda_{\text {exc }}\right)=563 \mathrm{~nm}$ ) of SP-NG-1 (black) and ME-NG-1 (red). Spectra were recorded on the solutions in DMSO $\left(1 \mathrm{~g} \mathrm{~L}^{-1}\right)$ with a $10 \mathrm{~mm}$ optical path length. d) $R_{\mathrm{h}}$ distribution of NG-1 determined by DLS analysis. Insert is the autocorrelation function $\left(\mathrm{g}^{(2)}\right.$ $-1)$ of NG-1 solution $\left(1 \mathrm{~g} \mathrm{~L}^{-1}\right)$ in PBS $(\mathrm{pH}=7.2)$ at $25^{\circ} \mathrm{C}$ measured at $90^{\circ}$. e) TEM images of NG-1. f) TEM images of SP-NG-1. Inserts are zoomed images (left) and histograms of the radius distributions obtained by statistical analyses for randomly selected NG-1 and SP-NG-1 particles from the TEM images by using ImageJ open-source software. 


\section{WILEY-VCH}

(a)

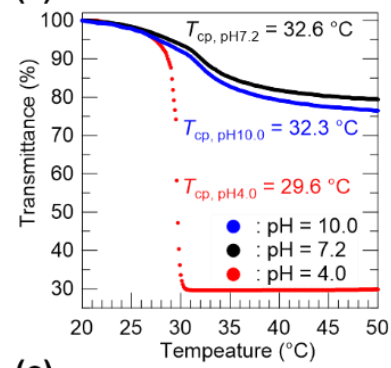

(b)

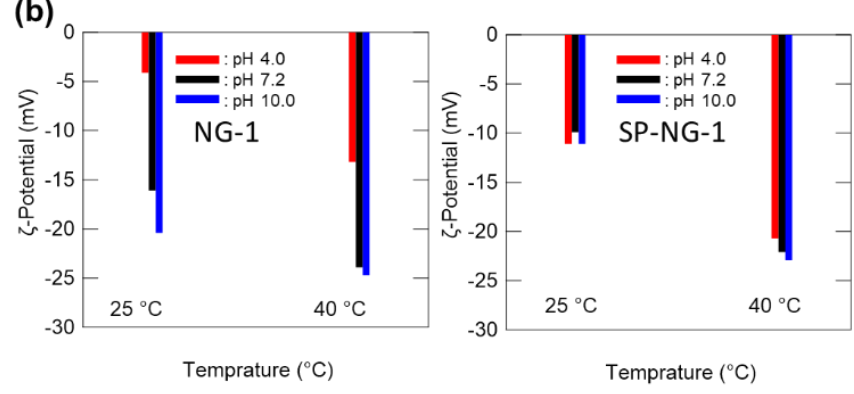

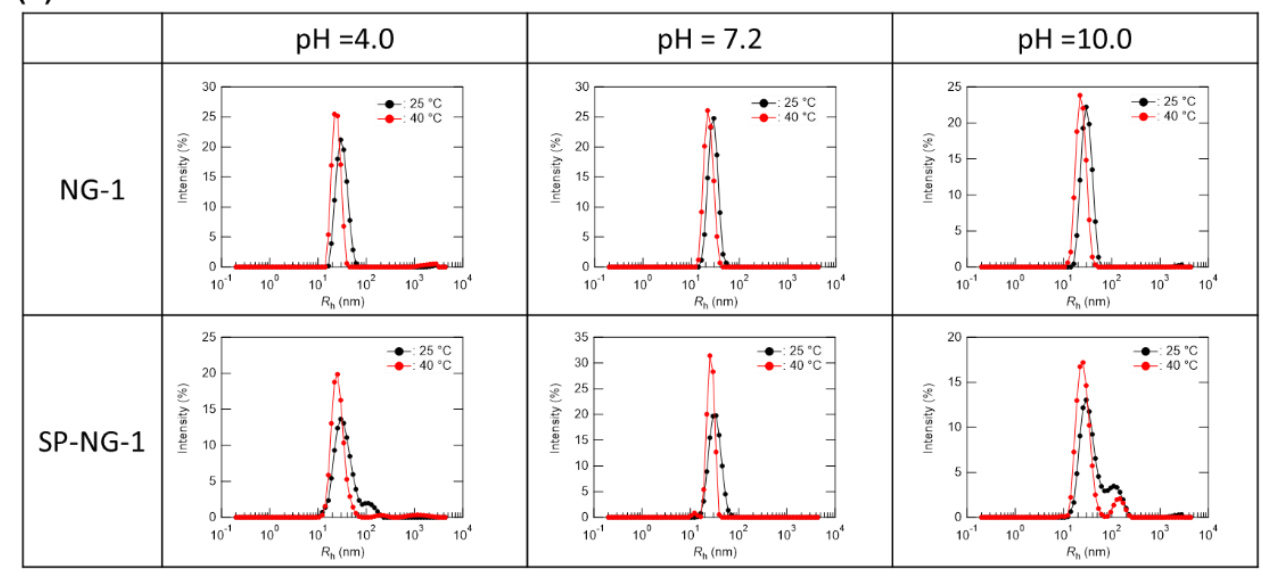

Figure 3. a) Temperature dependence of light $(\lambda=550 \mathrm{~nm})$ transmittance of NG-1 solution in PBS $\left(1 \mathrm{~g} \mathrm{~L}^{-1}\right)$ at $\mathrm{pH}=4.0$ (red), 7.2 (black), and 10.0 (blue). The cloud point temperature $\left(T_{\mathrm{cp}}\right)$ was defined as the temperature corresponding to the mid-point of total transmittance change between $20^{\circ} \mathrm{C}$ and $50{ }^{\circ} \mathrm{C}$. Transmittance (\%) was normalized as $100 \%$ at maximum. b) Thermal variation of the $\zeta$-potential of NG-1 (left) and SP-NG-1 (right) dispersions in PBS $\left(1 \mathrm{~g} \mathrm{~L}^{-1}\right)$ at different $\mathrm{pH}$. c) The $R_{\mathrm{h}}$ distributions of NG-1 and SP-NG-1 in PBS $\left(1 \mathrm{~g} \mathrm{~L}^{-1}\right)$ at different $\mathrm{pH}$ and temperature determined by DLS analysis. 


\section{WILEY-VCH}

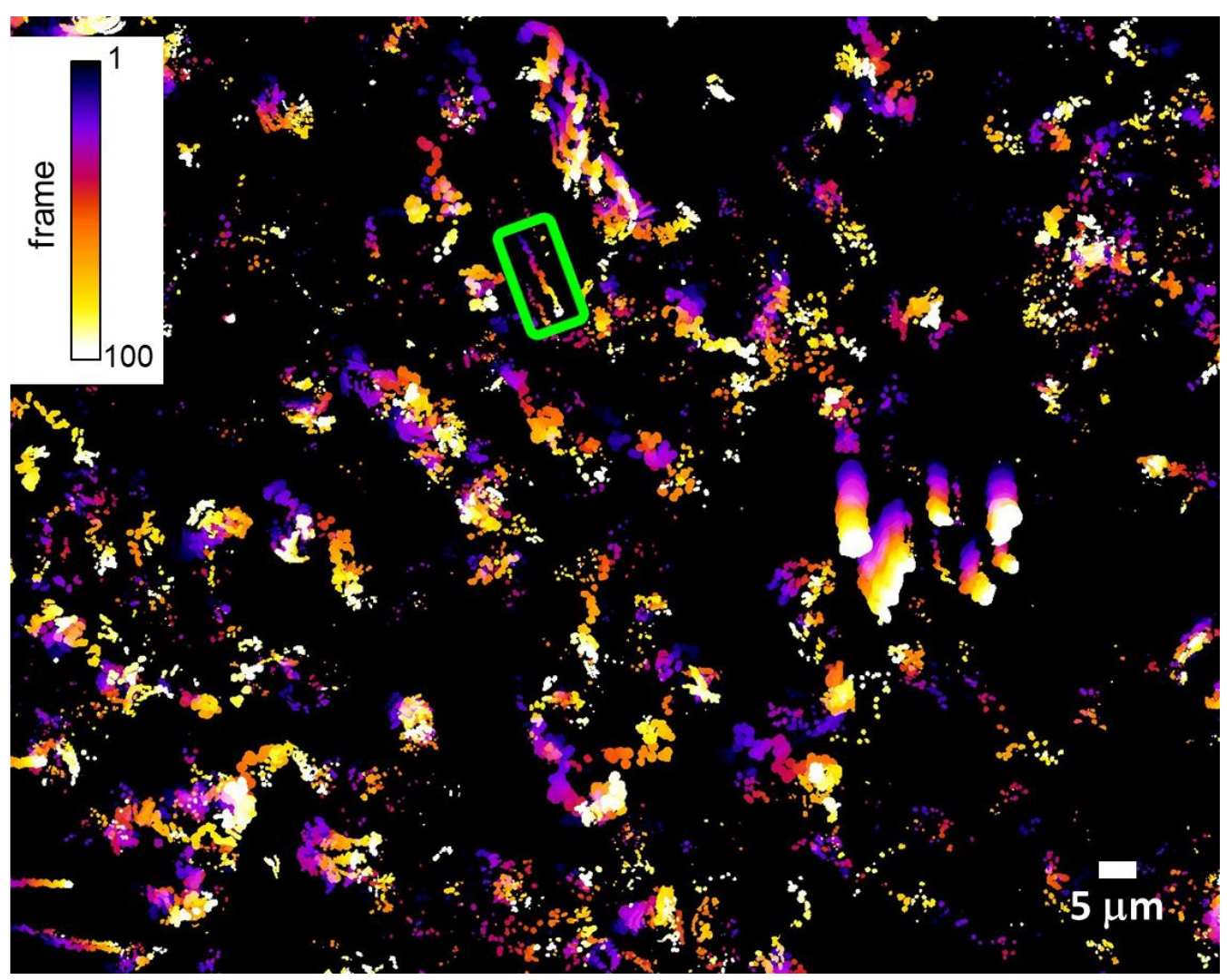

Figure 4. Trajectories of the moving microgels composed of SP-NG-1 in PBS $\left(5 \mathrm{~g} \mathrm{~L}^{-1}\right)$ at $\mathrm{pH}$ $=7.2$. The trajectories are composed of a sequence of 100 frames of microscopic pictures taken with the exposure time of $200 \mathrm{~ms}$ and the time interval between each frames of $640 \mathrm{~ms}$. 


\section{WILEY-VCH}

\section{Table of Contents:}

\section{Text for the Table of Contents:}

Active colloids that are drawn into directional collective motion through light, and respond also to changes of $\mathrm{pH}$ and/or temperature are reported. They are obtained by polymerization-induced thermal self-assembly of poly-( $N$-isopropylacrylamide) in the presence of poly(methacrylic acid) fragments. This approach generates $\mathrm{pH}$ - and temperature-responsive nanogels, which are surface-functionalized with spyropyran photochromic moieties that drive collective motion towards an applied UV-light.

Keywords: active matter, multi-stimuli-responsive, nano/microgels, collective motion, selfassembly

Authors: I. Otsuka, ${ }^{*}$ X. Zhang, F. M. Winnik*

Title: Phototropic Multiresponsive Active Nanogels

Graphic for the Table of Contents:

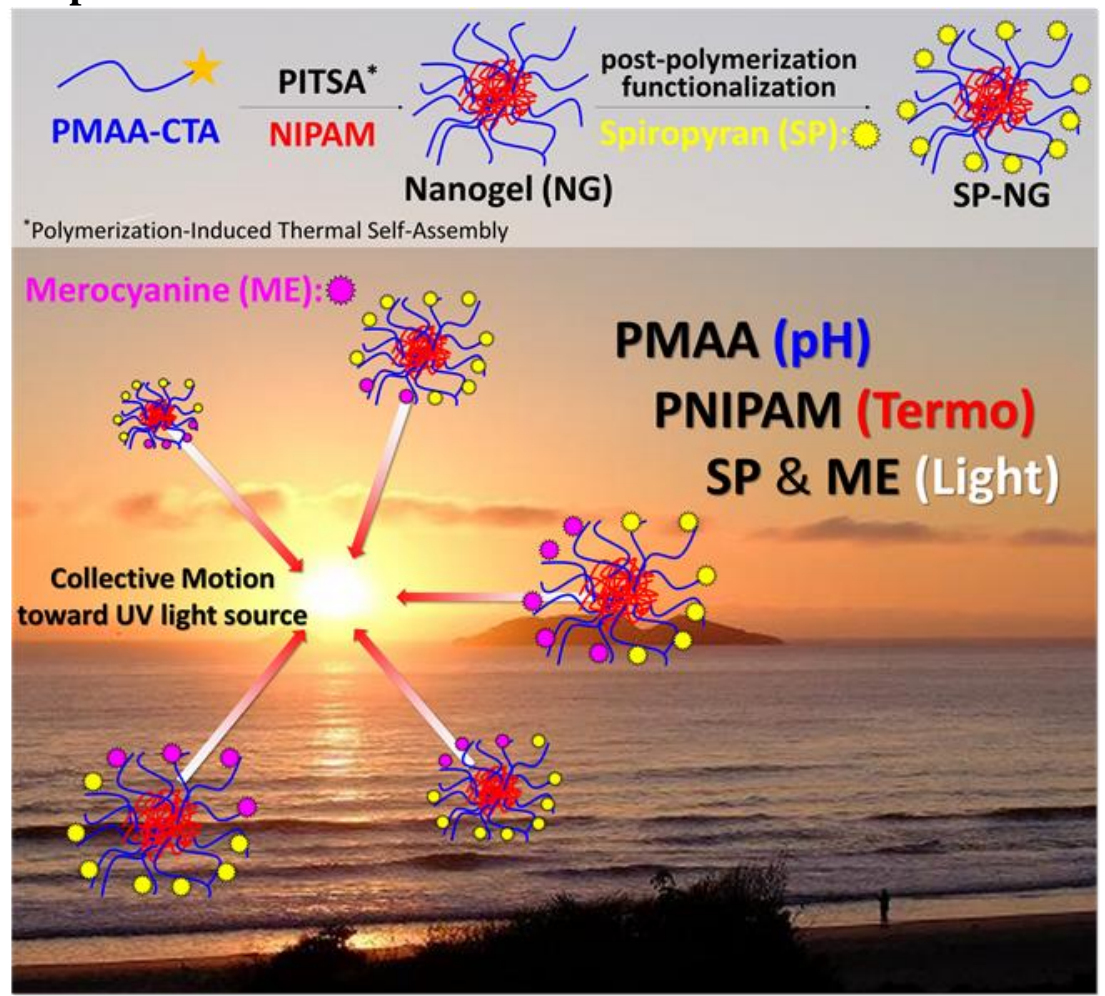

